\title{
When will we observe binary black holes precessing?
}

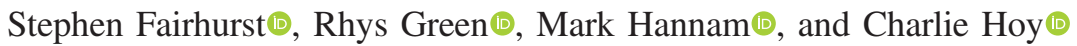 \\ School of Physics and Astronomy, Cardiff University, Cardiff CF24 3AA, United Kingdom
}

(Received 9 August 2019; accepted 22 July 2020; published 27 August 2020)

\begin{abstract}
After eleven gravitational-wave detections from compact-binary mergers, we are yet to observe the striking general-relativistic phenomenon of orbital precession. Measurements of precession would provide valuable insights into the distribution of black-hole spins, and therefore into astrophysical binary formation mechanisms. Using our recent two-harmonic approximation of precessing-binary signals [S. Fairhurst et al., Phys. Rev. D 102, 024055 (2020)], we introduce the "precession signal-to-noise ratio", $\rho_{p}$. We demonstrate that this can be used to clearly identify whether precession was measured in an observation (by comparison with both current detections and simulated signals), and can immediately quantify the measurability of precession in a given signal, which currently requires computationally expensive parameter-estimation studies. $\rho_{p}$ has numerous potential applications to signal searches, source-property measurements, and population studies. We give one example: assuming one possible astrophysical spin distribution, we predict that precession has a one in $\sim 25$ chance of being observed in any detection.
\end{abstract}

DOI: 10.1103/PhysRevD.102.041302

\section{INTRODUCTION}

The Advanced Laser Interferometer Gravitational-Wave Observatory (aLIGO) [1] and Advanced Virgo (AdV) [2], provide a unique method of observing mergers of black holes and/or neutron stars. Observations to date already provide insights into the mass and spin distributions of black holes $[3,4]$.

One important general relativistic effect that has not yet been observed is orbital precession. This arises when the black-hole spins are not aligned with the binary's orbital angular momentum. In contrast to Newtonian mechanics, where all angular momenta are individually conserved, in general relativity the binary's total angular momentum is (approximately) conserved, and the orbital angular momentum (and hence the orbital plane) and spins precess around it $[5,6]$. This leads to modulations in the amplitude and phase of the gravitational wave $(\mathrm{GW})$ signal. These are in general small effects and, in addition, whether they can be measured depends not only on the black-hole masses and spin magnitudes and directions, but also on the binary's orientation relative to the detector, and the observed GW polarization. For this reason, until now there was no straightforward way to determine how significantly precession would be imprinted onto a given waveform. The usual approach is to perform computationally expensive Bayesian analyses (see e.g., [3,7]), but even then, the misaligned spin components (which signify whether the binary is precessing) are degenerate with other parameters, and do not provide a direct measure of precession features in the signal. This makes it difficult to infer the impact of precession measurements on the properties of astrophysical binary populations and their formation mechanisms.

\section{THE TWO-HARMONIC APPROXIMATION}

We take advantage of a new characterization of precession, where the gravitational waveform for a precessing binary can be expressed as the sum of five harmonics, which form a power series in

$$
b=\tan (\beta / 2)
$$

where $\beta$ is the opening angle between total and orbital angular momentum [8]. Large values of $\beta$ are possible only when the in-plane component of spin, $\chi_{p}$ [9] is large; the mass ratio of the system is large, or there is a significant spin component antialigned with the orbital angular momentum-both of which serve to reduce the relative magnitude of the orbit-aligned angular momentum. For most configurations $b \ll 1$, and we may restrict attention to the first two harmonics and ignore terms of order $b^{2}$ or higher. It is only for binaries with $\beta \geq 45^{\circ}$, or equivalently $b \geq 0.4$, where the remaining harmonics become significant, and the two-harmonic approximation breaks down.

We may now write the observed waveform as a function of GW frequency as,

$$
h_{2 \text { harm }}(f) \approx \mathcal{A}_{0} h^{0}(f)+\mathcal{A}_{1} h^{1}(f),
$$

where $\mathcal{A}_{0}$ and $\mathcal{A}_{1}$ are complex, orientation dependent amplitudes and $h^{0}(f)$ and $h^{1}(f)$ are the waveforms of the two harmonics. Full details of the two-harmonic approximation are given in Ref. [8]. What is relevant to the current work is that the individual harmonics do not exhibit precession modulations; these show up due to beating between the harmonics, whose respective dephasing can 
be related to the frequency with which the orbital angular momentum precesses around the total angular momentum. The amplitudes $\mathcal{A}_{0}$ and $\mathcal{A}_{1}$ depend on the binary's orientation and polarization, but are approximately constant throughout a given signal.

\section{OBSERVABILITY OF PRECESSION}

Since the individual harmonics are indistinguishable from nonprecessing waveforms, it is only when two precession harmonics can be independently observed that precession can be unambiguously identified. For precession to be observable, we therefore require that the expected signal-to-noise ratio (SNR) in both of the harmonics is above some threshold.

The expected SNR for a signal $h$ embedded in data from a detector with a noise power spectral density $S(f)$ is given as $\hat{\rho}^{2}=(h \mid h)$, where the inner product is,

$$
(g \mid h)=4 \operatorname{Re} \int_{f_{o}}^{f_{\max }} \frac{g(f) h^{\star}(f)}{S(f)} d f,
$$

and ${ }^{\star}$ denotes complex conjugation [10]. In cases with more than one precession cycle between $f_{o}$ and $f_{\max }$, the two harmonics will be close to orthogonal, $\left(h^{0} \mid h^{1}\right) \approx 0$, and the precession SNR is simply defined as the expected SNR in the weaker harmonic:

$$
\rho_{p}=\operatorname{Min}\left(\left|\mathcal{A}_{0} h^{0}\right|,\left|\mathcal{A}_{1} h^{1}\right|\right),
$$

where $|h|:=\sqrt{(h \mid h)}$. When there is less than one precession cycle, it is necessary to also subtract the power aligned with the dominant harmonic, as described in detail in Ref. [8], before determining whether the second harmonic is observable.

It remains to determine a threshold above which $\rho_{p}$ can be considered as evidence for precession. Consider the situation where an event has been observed, so there is significant SNR in at least one harmonic. In the absence of measurable precession, and assuming well-modelled Gaussian noise, the SNR in the second harmonic will be $\chi^{2}$ distributed with two degrees of freedom, where the two degrees of freedom correspond to the real and imaginary parts of the complex amplitude. Therefore, in the absence of precession, $\rho_{p}>2.1$ is expected in less than $10 \%$ of cases, and $\rho_{p}>3$ in approximately $1 \%$ of cases. We therefore use these simple thresholds as a measure of the strength of evidence for observable precession. ${ }^{1}$

In Fig. 1 we show the recovered distribution of $\chi_{p}$ and $\rho_{p}$ for a number of signals, both real and simulated. For each

\footnotetext{
${ }^{1}$ A more detailed analysis would consider the volume of the binary parameter space consistent with the nonprecessing and precessing parameters and use these to appropriately weight the likelihoods of the precessing and nonprecessing signals. This will have some impact on the required $\rho_{p}$ threshold, but is unlikely to change it significantly.
}

signal, we use a nested sampling routine within the LALInference code $[7,11]$ to obtain posterior probability distributions for the parameters. This is the same infrastructure that was used to measure the properties of the LIGO-Virgo observations, and we present our results in the same form as in, for example, the GWTC-1 catalogue [3], by using the PESummary library [12]. The new feature is our calculation of $\rho_{p}$.

First, we show the recovered $\chi_{p}$ and $\rho_{p}$ distributions for a set of simulated signals, generated using the IMRPhenomPv2 model [13], each with the same choices of masses and spins-total mass $M=40 M_{\odot}$, mass ratio $2: 1$, and an in-plane spin of $\chi_{p}=0.4$ on the large black hole only-but varying orientation, encoded by the angle $\theta$ between the total angular momentum and the line of sight. The distance to each signal is chosen to ensure a fixed expected SNR of 20 in the aLIGO detectors at the sensitivity of the second observing run $(\mathrm{O} 2)$ [3], resulting in a distance variation by a factor of $\approx 3.5$ between the least and most inclined systems.

For binaries with total angular momentum closely aligned with the line of sight, $\theta<45^{\circ}$, the precessing SNR is consistent with no power in the $h^{1}$. The posterior on $\chi_{p}$ is consistent with the prior at low $\chi_{p}$ but excludes $\chi_{p} \gtrsim 0.7$. When $\theta>45^{\circ}$, the angular momentum is significantly misaligned with the line of sight and there is significant power in both harmonics, leading to a value of $\rho_{p}$ inconsistent with noise alone and little support for values of $\chi_{p} \lesssim 0.1$. However, using $\chi_{p}$ alone, even after performing the parameter recovery there are no simple criteria to determine when precession is observed. A natural choice might require that the $90 \%$ confidence interval for $\chi_{p}$ exclude zero, but this will always be the case, primarily due to the shape of the prior. Furthermore, even though we know all of the parameters a priori, it is impossible to determine whether precession will be observable without generating the waveform and performing the parameter recovery.

The precession SNR solves these problems. A value of $\rho_{p}>2.1$ tells us immediately that there is evidence of observable precession. Most significantly, the expected precession SNR $\rho_{p}$ (red lines on the middle plot) can be calculated directly from the signal parameters; no detailed parameter estimation analysis is necessary. Thus, for the first time, we are able to identify immediately whether precession would be measurable in a given configuration. We see in Fig. 1 that for each inclination, the true value for $\rho_{p}$ lies within the recovered $90 \%$ credible interval however the posterior is not centered around the true value. This is due to selection and prior effects. In Ref. [14], we investigate these selection effects as well as providing a detailed exploration of the observability of precession over the parameter space of masses, spins and binary orientation. 

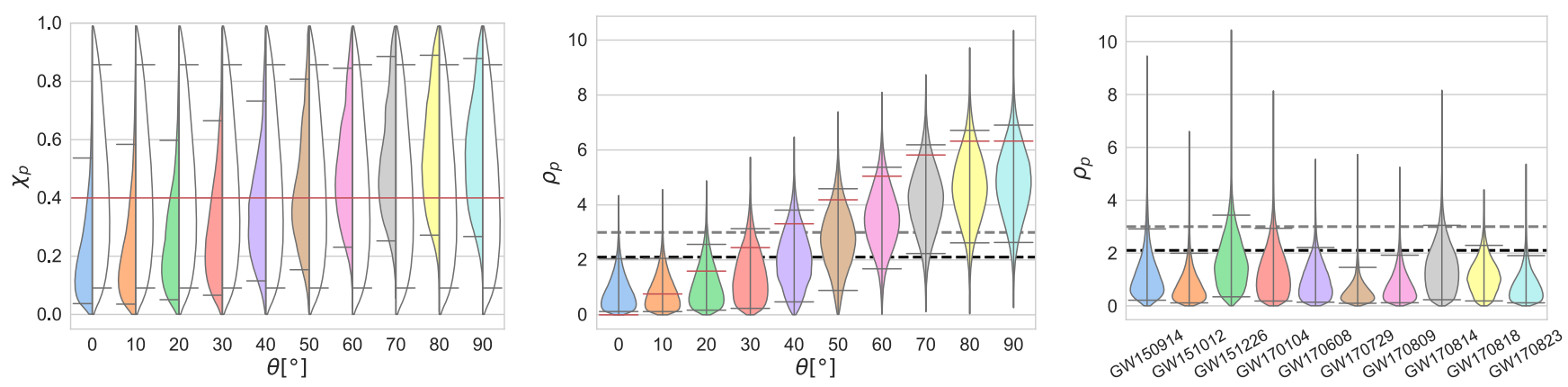

FIG. 1. For a set of simulated signals with fixed masses and spins (see text), we show the posterior and prior (white) distributions for $\chi_{p}$ (left), and posterior distributions for $\rho_{p}$ (middle) for a range of different binary orientations, $\theta$. The grey lines show the $90 \%$ confidence regions, the solid red lines show the true values of $\chi_{p}$ and $\rho_{p}$ respectively and the dashed black and grey lines indicates the thresholds for observable precession at $\rho_{p}=2.1$ and $\rho_{p}=3$. The right panel shows the $\rho_{p}$ distribution for the ten binary-black-hole observations in $\mathrm{O} 1$ and $\mathrm{O} 2[3]$.

Figure 1 also shows the distribution of $\rho_{p}$ for the $\mathrm{BBH}$ merger signals that were observed in the aLIGO and AdV $\mathrm{O} 1$ and $\mathrm{O} 2$ runs [3]. No evidence of precession was found in these signals [4], as is made clear from the recovery of $\rho_{p}$. There are several cases where the distribution extends to higher values, but the median never exceeds the 2.1 threshold. These results demonstrate the efficacy of $\rho_{p}$.

\section{WHEN WILL WE OBSERVE PRECESSION?}

We can use the observation of precession to distinguish different binary formation scenarios. The precession SNR makes it straightforward to perform an in-depth investigation of various models and identify the fraction of signals for which precession effects will be observable. Such a study was not previously possible, due to the difficulty in classifying observability of precession. Instead, limited investigations of the parameter space have been performed [15], or inferences of the distributions for the spin magnitudes and orientations obtained [16,17], again with a limited sample size.

We investigate nine astrophysically motivated populations of black hole binaries, comprised of three distributions of spin magnitude, and three distributions of spin orientation for the individual black holes in the binary. The spin-magnitude distributions are those used in Refs. [18-20]: low and high are triangular, peaked either at zero or extremal spin, and flat is a uniform distribution

TABLE I. The probability of observing precession, $\rho_{p}>3$, for an observed binary (standard text) from each spin distribution and the probability of not observing precession in 10 random draws (bold and italic) from each spin distribution

\begin{tabular}{lcccccc}
\hline \hline & \multicolumn{2}{c}{ Aligned } & \multicolumn{2}{c}{ Isotropic } & \multicolumn{2}{c}{ Precessing } \\
\hline Low & 0.043 & $\mathbf{0 . 6 4 4}$ & 0.151 & $\mathbf{0 . 1 9 4}$ & 0.173 & $\mathbf{0 . 1 5 0}$ \\
Flat & 0.077 & $\mathbf{0 . 4 4 8}$ & 0.276 & $\mathbf{0 . 0 4 0}$ & 0.327 & $\mathbf{0 . 0 1 9}$ \\
High & 0.105 & $\mathbf{0 . 3 3 1}$ & 0.354 & $\mathbf{0 . 0 1 3}$ & 0.412 & $\mathbf{0 . 0 0 5}$ \\
\hline \hline
\end{tabular}

between zero and one. The spin-orientation is characterized by the distribution for the angle $\sigma$ between each black hole's spin and the orbital angular momentum: aligned is a triangular distribution in $\cos \sigma$, which peaks at 1 and can take values $0.85<\cos \sigma<1.0,\left(\sigma \lesssim 30^{\circ}\right)$; precessing is triangular in $\cos \sigma$ peaked at 0 , with values $-0.15<\cos \sigma<0.15,\left(80^{\circ} \lesssim \sigma \lesssim 100^{\circ}\right)$; isotropic is uniform in $\cos \sigma$ between -1 and 1 . For each population, we generate $10^{5}$ binaries with masses drawn from a power law mass distribution with $p\left(m_{1}\right) \propto m^{-2.35}$, and $p\left(m_{2}\right)$ uniform in $m_{2}$ between $5 M_{\odot}$ and $m_{1}$ (as in [20]), and distributed uniformly in volume and binary orientation.

Table I shows the probability of observing precession in a single event drawn from each of the nine populations, observed with $\mathrm{O} 2$ sensitivity while assuming zero noise. For this study, we use the higher threshold of $\rho_{p}>3$, corresponding to a $1 \%$ false rate, to indicate strong evidence for observed precession. When observing a population of events, the number of events exceeding this threshold when there is no precession in the system remains low. ${ }^{2}$

As expected, we are most likely to observe precession when the black holes have high spins that lie preferentially in the orbital plane (high-precessing configurations) and least likely for black holes with low spins, or with spins preferentially aligned with the orbital angular momentum (low-aligned configurations). Given that precession has not been observed in GW detections to date, we are able to restrict the spin distribution. Table I shows the probability of detecting ten signals with no observable precession from each of the nine spin distributions. Based on precession measurements alone, we strongly disfavor all precessing distributions. Although these are already considered

\footnotetext{
${ }^{2}$ As our analysis assumes zero noise, the fraction of binaries with observable precession will be slightly underestimated. At a threshold of $\rho_{p}>2.1$, the effect would be significant while at a threshold of $\rho_{p}>3$, the difference is small.
} 

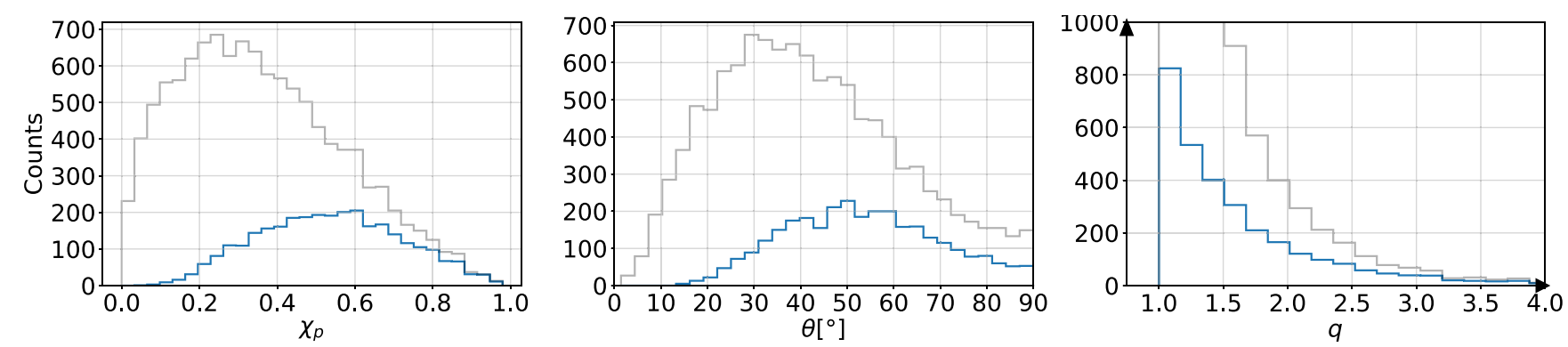

FIG. 2. The distribution of $\chi_{p}, \theta$ and $q$ for observable binaries (grey), and those with measurable precession (blue), assuming a low isotropic spin distribution. $\theta$ is the inclination angle folded to $[0, \pi / 2]$. The y-axis labels the number of observed events in each bin, out of $10^{5}$ simulated signals with low isotropic spins.

astrophysically unlikely, there are models that predict preferentially in-plane spins $[21,22]$. We also disfavor isotropic spins with flat or high magnitudes. Thus, the lack of observed precession points towards low spins, or spins preferentially aligned with the orbital angular momentum.

Previous constraints on spins have primarily been provided by considering the measurable aligned-spin component [3,18-20] and provide strong evidence against all but low aligned or iostropic distributions, with low isotropic spins preferred. Combining the aligned spin and precession results will further restrict the spin distribution consistent with GW observations, and will likely require spin magnitudes even smaller than our low distribution (see also Ref. [4]).

\section{WHERE WILL WE OBSERVE PRECESSION?}

We are able to identify, for the first time, the regions of parameter space that lead to signals with observable precession. In Fig. 2, we show the expected distribution of the precessing spin $\chi_{p}$, binary orientation $\theta$ and mass ratio $q$ for observable binaries and binaries with observable precession, $\rho_{p}>3$, assuming a low isotropic distribution of spins. We identify clear regions of the parameter space where precession is more likely to be observed: large values of $\chi_{p}$, binaries that are close to edge-on, $\theta>45^{\circ}$, and systems with high mass ratio. Regions where the chance of observing precession is close to zero include binaries with $\chi_{p}<0.2$ or where the total angular momentum is within $20^{\circ}$ of the line of sight. These results are consistent with expectations based upon smaller studies using detailed parameter estimation techniques [15]. We also note that most observations of precession will be in comparablemass binaries, i.e., $q \leq 2$. This is a surprising, new result. It is well known that precession is more easily measured at higher mass ratios [5], which is confirmed by our study: precession is observed in $<12 \%$ of detections with $q<2$, but $>35 \%$ for $q>2$. However, with $\sim 90 \%$ of observations expected to have $q<2$, these vastly outnumber the highermass-ratio observations, and we find that $\sim 75 \%$ of precession observations will come from detections of binaries with $q<2$.

\section{DISCUSSION}

In this paper we have used a simple method to identify when precession is measurable in a compact binary GW signal. The gravitational waveform is well approximated by the first two harmonics in a power series expansion in the tangent of the half-opening angle [8], and the unambiguous observation of precession requires the identification of both of these harmonics in the data. The precession $\operatorname{SNR} \rho_{p}$ is a simple measure of this observability. We have demonstrated the efficacy of $\rho_{p}$ through parameter estimation studies and also provided the distributions of $\rho_{p}$ for the aLIGO-AdV observations to date. Using our definition of precession SNR, we have identified how often precession will be observed for a variety of potential astrophysical spin distributions. For the most likely distribution, based on current observations (low-aligned) there is a $83 \%$ chance that precession will be measured after $\sim 40$ observations, and is therefore likely to be observed during the current third aLIGO-AdV observing run (O3). The nonmeasurement of precession by the end of $\mathrm{O} 3$ would place much stronger constraints on spin orientations and magnitudes.

The precession SNR has many applications. Most immediately, it allows us to determine the measurability of precession in a system without performing computationally expensive parameter estimation. This allows us to, e.g., easily fold precession information into population analyses of black-hole binaries. In future work, we will explore whether the value of $\rho_{p}$ can be used to predict the measured $\chi_{p}$ distribution. The precessing SNR also gives us a simple way to identify regions of the parameter space where precession is important, a necessary first step in extending existing GW searches to explicitly use precessing waveforms [23].

\section{ACKNOWLEDGMENTS}

We thank Eleanor Hamilton, Ian Harry, Alistair Muir, Francesco Pannarale, Vivien Raymond, Cory Thomas, and 
Vaibhav Tiwari for discussions. This work was supported by Science and Technology Facilities Council (STFC) Grant No. ST/L000962/1 and European Research Council Consolidator Grant No. 647839. Computational resources were provided by Cardiff University, funded by STFC support for UK involvement in aLIGO operations.

Note added.-Since the first submission of this paper, $\rho_{p}$ has been used in the analysis of the aLIGO-AdV signals GW190412 [24] and GW190814 [25].
[1] J. Aasi et al., Advanced LIGO, Classical Quantum Gravity 32, 115012 (2015).

[2] F. Acernese et al., Advanced Virgo: A second-generation interferometric gravitational wave detector, Classical Quantum Gravity 32, 024001 (2015).

[3] B. P. Abbott et al., GWTC-1: A Gravitational-Wave Transient Catalog of Compact Binary Mergers Observed by LIGO and Virgo during the First and Second Observing Runs, Phys. Rev. X 9, 031040 (2019).

[4] B. P. Abbott et al., Binary black hole population properties inferred from the first and second observing runs of Advanced LIGO and Advanced Virgo, Astrophys. J. 882, L24 (2019).

[5] T. A. Apostolatos, C. Cutler, G. J. Sussman, and K. S. Thorne, Spin induced orbital precession and its modulation of the gravitational wave forms from merging binaries, Phys. Rev. D 49, 6274 (1994).

[6] T. A. Apostolatos, Search templates for gravitational waves from precessing, inspiraling binaries, Phys. Rev. D 52, 605 (1995).

[7] J. Veitch et al., Parameter estimation for compact binaries with ground-based gravitational-wave observations using the LALInference software library, Phys. Rev. D 91, 042003 (2015).

[8] S. Fairhurst, R. Green, C. Hoy, M. Hannam, and A. Muir, Two-harmonic approximation for gravitational waveforms from precessing binaries, Phys. Rev. D 102, 024055 (2020).

[9] P. Schmidt, F. Ohme, and M. Hannam, Towards models of gravitational waveforms from generic binaries II: Modelling precession effects with a single effective precession parameter, Phys. Rev. D 91, 024043 (2015).

[10] B. Allen, W. G. Anderson, P. R. Brady, D. A. Brown, and J. D. E. Creighton, FINDCHIRP: An algorithm for detection of gravitational waves from inspiraling compact binaries, Phys. Rev. D 85, 122006 (2012).

[11] J. Veitch and A. Vecchio, Bayesian coherent analysis of inspiral gravitational wave signals with a detector network, Phys. Rev. D 81, 062003 (2010).

[12] C. Hoy and V. Raymond, PESummary: The code agnostic parameter estimation summary page builder, arXiv: 2006.06639.

[13] M. Hannam, P. Schmidt, A. Bohé, L. Haegel, S. Husa, F. Ohme, G. Pratten, and M. Pürrer, Simple Model of
Complete Precessing Black-Hole-Binary Gravitational Waveforms, Phys. Rev. Lett. 113, 151101 (2014).

[14] R. Green et al., Identifying when precession can be measured in gravitational waveforms (to be published).

[15] S. Vitale, R. Lynch, J. Veitch, V. Raymond, and R. Sturani, Measuring the Spin of Black Holes in Binary Systems Using Gravitational Waves, Phys. Rev. Lett. 112, 251101 (2014).

[16] D. Wysocki, J. Lange, and R. O'Shaughnessy, Reconstructing phenomenological distributions of compact binaries via gravitational wave observations, Phys. Rev. D 100, 043012 (2019).

[17] C. Talbot and E. Thrane, Determining the population properties of spinning black holes, Phys. Rev. D 96, 023012 (2017).

[18] W. M. Farr, S. Stevenson, M. C. Miller, I. Mandel, B. Farr, and A. Vecchio, Distinguishing spin-aligned and isotropic black hole populations with gravitational waves, Nature (London) 548, 426 (2017).

[19] B. Farr, D. E. Holz, and W. M. Farr, Using Spin to understand the formation of LIGO and Virgos black holes, Astrophys. J. 854, L9 (2018).

[20] V. Tiwari, S. Fairhurst, and M. Hannam, Constraining blackhole spins with gravitational wave observations, Astrophys. J. 868, 140 (2018).

[21] F. Antonini, C. L. Rodriguez, C. Petrovich, and C. L. Fischer, Precessional dynamics of black hole triples: Binary mergers with near-zero effective spin, Mon. Not. R. Astron. Soc. 480, L58 (2018).

[22] C. L. Rodriguez and F. Antonini, A triple origin for the heavy and low-spin binary black holes detected by LIGO/ Virgo, Astrophys. J. 863, 7 (2018).

[23] I. Harry, S. Privitera, A. Boh, and A. Buonanno, Searching for gravitational waves from compact binaries with precessing spins, Phys. Rev. D 94, 024012 (2016).

[24] R. Abbott et al., GW190412: Observation of a binaryblack-hole coalescence with asymmetric masses, arXiv: 2004.08342.

[25] R. Abbott et al., GW190814: Gravitational waves from the coalescence of a 23 solar mass black hole with a 2.6 solar mass compact object, Astrophys. J. 896, L44 (2020). 